\title{
Cartographic Applications Software
}

The Office of the Assistant Division Chief for Research, National Mapping Division, develops computer software for the solution of geometronic problems in the fields of surveying, geodesy, remote sensing, and photogrammetry. Software that has been developed using public funds is available on request for a nominal charge to recover the cost of duplication.

The complete program package includes IBM Job Control Language (JCL) to compile and execute the program and FORTRAN source code on a 9-track magnetic tape. A user's manual is included unless otherwise noted. Also included is a copy of the computer listed output of the contents of the tape. The computer listed output (including a compiler listing of the source code) and user documentation may be purchased at a reduced rate without the magnetic tape file.

All programs on this list, unless otherwise noted, are written in FORTRAN. Some programming modification may be necessary to compile and optimize the code for the target FORTRAN compiler.

USGS data tapes and 5 1/4-inch diskettes are produced on computer equipment that is periodically tested and adjusted to conform to industry standards. However, there is no guarantee they will read on other computer equipment.

If a customer has difficulty using a tape or diskette, it may be returned to the Earth Science Information Center (ESIC) and a reasonable effort will be made to replace it. Because the programs are not protected by a copyright, no refunds can be made for returned tapes or diskettes.

For additional information, contact any ESIC or call 1-800-USA-MAPS.

\section{Software products}

Linear Horizontal Transformation with

Linear and Affine Options (H253)-

Performs a linear least-square adjustment of measured $X-Y$ coordinates to true or known coordinates. Points may also be transformed to the true system from the observed, or vice versa. The adjustment may be affine, separating the $X$ and $Y$ parameters. FORTRAN source code has 581 lines.

SIMBA (Simultaneous Block Adjustment of Models) (H255)-Simultaneously adjusts photogrammetric units to each other and to ground control. SIMBA allows up to 100 units. The input units may be models observed semianalytically on a stereoplotter or developed analytically from comparator observations. FORTRAN source code has 1,621 lines.

Complete Comparator Calibration (H290)-Performs the calibration necessary for the quality control of measurements made on precise comparators. Written by Lawrence Fritz of the National Ocean Survey, this program is documented as NOAA Technical Report NOS 57 (July 1973) available from the National Technical Information Service, Springfield, Virginia. A procedure manual on Calibration of Comparators is supplied in lieu of a user's manual or software documentation. FORTRAN source code has 2,828 lines.

\section{Universal Transverse Mercator} Conversion (J380)-Converts geodetic coordinates to Universal Transverse Mercator (UTM) X-Y coordinates or vice versa with the option to force to a particular UTM zone. The program will also convert UTM coordinates in one zone to the UTM values for an adjacent zone. Software documentation is included. FORTRAN source code has 447 lines.
Generalized Plane Coordinate Conversion Subroutine Package (PLANE)-A group of FORTRAN subroutines used to perform grid coordinate conversion between geographic coordinates (latitude, longitude) and the appropriate rectangular system (State Plane Coordinate System or UTM). Both forward and inverse computations may be performed. Requires a main or driver program. FORTRAN source code has 1,135 lines.

TESDEM (K373)—Compares digital elevation models (DEM's) in the standard USGS format against test points with known X-Y-Z coordinates to evaluate the accuracy of the DEM. The results are inserted into Record Type C for the DEM. PLANE subroutine package and software documentation are included. FORTRAN source code has 797 lines for TESDEM and 1,135 lines for PLANE.

GRIDEM (K374)—Standard USGS-format DEM's in a gridded (not random) pattern can be regridded (resampled) to a different interval, orientation, or projection (State Plane or UTM). PLANE subroutine package and software documentation are included. FORTRAN source code has 1,106 lines for K374 and 1,135 lines for PLANE.

ORION (K394)_Focuses on the value of studying the Earth's surface with aerial photographs and discusses applications such as studying seasonal changes in vegetation or wildlife habitat and evaluating damage caused by geologic hazards like volcanoes, landslides, and floods. PLANE subroutine package and software documentation are included. FORTRAN source code has 969 lines for K394 and 1,135 lines for PLANE. 
X-Y to Latitude-Longitude and Grid Coordinates (K665)-Performs the transformation of Cartesian coordinates collected in an arbitrary system, such as from a digitizer, to latitude-longitude and $X-Y$ coordinates in any State Plane Coordinate System or UTM zone. PLANE subroutine package and software documentation are included. FORTRAN source code has 368 lines for K665 and 1,135 lines for PLANE.

\section{Generalized Plane Coordinate}

Transformation (K714)-Converts coordinate data input in any State Plane Coordinate System zone, UTM zone, or geographic coordinates to output in another zone or geographic coordinates. Input and output formats and data locations are defined at execution time and the program is designed to be executed in batch mode. PLANE subroutine package and software documentation are included. FORTRAN source code has 1,540 lines and 1,135 lines for PLANE.

Interactive Grid Coordinates Conversion (K759)-Similar to K714, this program's computations are performed interactively on a time-sharing computer system. PLANE subroutine package and software documentation are included. FORTRAN source code has 289 lines for K759 and 1,135 lines for PLANE.

GS-CAM 1 -Geological Survey Cartographic Automated Mapping (K971)-The USGS has modified the CIA program CAM, Version 5, to use the General Cartographic Transformation Package (GCTP) for projection transformations. In addition, various corrections have been applied and additional flexibility in the use of data files, such as World Data Bank II, has been added. The documentation has been significantly upgraded. FORTRAN source code has 16,472 lines.

Interactive Grid Coordinate Conversion for Personal Computers (PLANE-PC)Performs the conversion of geographic coordinates to a State Plane Coordinate System or to UTM coordinates, or vice versa. The computations are performed interactively on a microprocessor-based computer system such as an IBM PC or IBM compatible PC. A 5 1/4-diskette containing tables and 1,040 lines of FORTRAN source code are included.
General Cartographic Transformation Package (GCTPV2)-A system of FORTRAN subroutines that permits the transformation of coordinate pairs from one of 23 map projections to another. It will perform State Plane Coordinate transformations on either NAD 1927 or NAD 1983. It is distributed with driver programs L176V2 and J898V2. This is the standard computer software used by the National Mapping Division for map projection computations. Software documentation is supplied. FORTRAN source code has 5,431 lines.

GCTP Driver Program (J898V2)Converts geographical coordinates to one of 21 projection coordinates, then back to geographical coordinates to analyze the input and output differences. FORTRAN source code has 1,898 lines. J898V2 is distributed as part of the GCTP package.

General Map Projection Transformation (L176V2)-The General Cartographic Transformation Package has been augmented by a driver program to permit the batch transformation of coordinates from one of 23 projection systems to another with any one of 20 spheroids selected for input and output to the package. FORTRAN source code has 1,375 lines. L176V2 is distributed as part of the GCTP package.

Geodetic Position and Inverse (W5501)Solves for either the geodetic position or the inverse problem in any quadrant of the Earth using the parameters of one of five commonly used ellipsoids. Observations at the stations are used to compute positions and back-azimuths. FORTRAN source code and data have 321 lines.

\section{General Integrated Analytical}

Triangulation (GIANT)-Performs a leastsquare adjustment of frame photographs that are constrained or arbitrarily arranged or both. The adjustment will propagate input error estimates to the computed parameters. The program is written in FORTRAN to run on the ENCORE 32/9780 series of computers under the Mapped Programming Executive (MPX) operating system. FORTRAN source code and data have 6,354 lines. 